REVIEW ARTICLE

\title{
Perspectives on diabetic retinopathy from advanced retinal vascular imaging
}

\author{
Janice X. Ong (iD ${ }^{1}$ and Amani A. Fawzi (iD) ${ }^{1 凶}$ \\ (c) The Author(s), under exclusive licence to The Royal College of Ophthalmologists 2021
}

\begin{abstract}
Diabetic retinopathy (DR) is a microvascular complication of diabetes and the most common cause of acquired vision loss in adults worldwide. DR is associated with long-term chronic hyperglycaemia and its detrimental effects on the neurovascular structure and function of the retina. Direct imaging of the retinal vasculature and staging of DR has been traditionally based on fundoscopy and fluorescein angiography, which provide only 2D views of the retina, and in the case of fluorescein angiography, requires an invasive dye injection. In contrast, advanced retinal imaging modalities like optical coherence tomography angiography (OCTA) and adaptive optics (AO) are non-invasive and provide depth-resolved, 3D visualization of retinal vessel structure as well as blood flow. Recent studies utilizing these imaging techniques have shown promise in evaluating quantitative vascular parameters that correlate tightly to clinical DR staging, elucidating functional changes in early diabetes, and monitoring DR treatment response. In this article, we discuss and synthesize the results of advanced retinal imaging studies in DR and their implications for our clinical and pathophysiologic understanding of the disease. Based on the recent literature, we also propose a model to describe the differential changes in vascular structure and flow that have been described on advanced retinal imaging as DR progresses. Future studies of these imaging modalities in larger and more diverse populations, as well as corroboration with histological and functional studies, will be important to further our understanding of DR.
\end{abstract}

Eye (2022) 36:319-327; https://doi.org/10.1038/s41433-021-01825-2

\section{INTRODUCTION}

Diabetic retinopathy (DR) is a microvascular complication of diabetes and one of the leading causes of blindness in patients aged 20-74 [1]. DR is estimated to affect one-third of the over 460 million diabetic patients worldwide [2]. As a progressive and vision-threatening condition, its late-stage complications including vitreous haemorrhage, traction retinal detachment, neovascular glaucoma, and vision loss [3,4]. DR is also associated with heightened risk of other microvascular and systemic complications of diabetes including nephropathy and cardiovascular disease $[5,6]$.

The pathophysiology of DR is characterized by chronic hyperglycaemia, which promotes a pro-inflammatory environment characterized by the accumulation of acute-phase reactants, advanced glycation end products, and reactive oxygen species [710]. On a histologic level, these changes result in early pericyte loss and endothelial damage [11, 12]. Capillary acellularity and capillary closure ensue, leading to the reduced blood flow and ischemia that characterize the earlier, non-proliferative stages of DR (NPDR) [13, 14]. Loss of endothelial integrity also leads to vascular leakage, vascular dilation to form telangiectasias, as well as microaneurysms, and haemorrhages [15]. Continued ischemia eventually drives a transition to a pro-angiogenic state with the upregulation of signalling molecules like vascular endothelial growth factor (VEGF), leading to the preretinal neovascularization that marks the onset of proliferative diabetic retinopathy (PDR) [16].
Optical coherence tomography angiography (OCTA) is a noninvasive imaging modality that allows depth-resolved imaging of the retinal structure and vasculature[17]. OCTA systems use multiple structural OCT scans of the same retinal location to extract motion and blood flow data, generating a map of vessel structure and flow that is depth-resolved $[18,19]$. Common vascular parameters that have been extracted from OCTA images include perimeter and area of the foveal avascular zone (FAZ); vessel density, measured as percentage of scan area occupied by vessels; and vessel length density, which skeletonizes vessels to a single pixel width to reduce the influence of large superficial vessels.

The exquisite depth resolution of OCTA, along with the development of segmentation methods that identify retinal layers based on landmarks from the corresponding structural OCT, have enabled clinicians to visualize individual layers of the retinal capillary network to a level of detail that was not possible before $[20,21]$. In contrast, fluorescein angiography, considered the gold standard for evaluating the retinal vasculature, requires an intravenous dye injection and results in $2 \mathrm{D}$ images that best visualize superficial vessels but fails to visualize the deeper vascular layers [18, 22].

\section{OCTA of the normal capillary network and neurovascular coupling}

The macular capillaries comprise a trilaminar vascular network composed of three histologically distinct capillary plexusessuperficial (SCP), middle (MCP), and deep (DCP)[23]. The radial

${ }^{1}$ Department of Ophthalmology, Feinberg School of Medicine, Northwestern University, Chicago, IL, USA. ${ }^{\circledR}$ email: afawzimd@gmail.com 


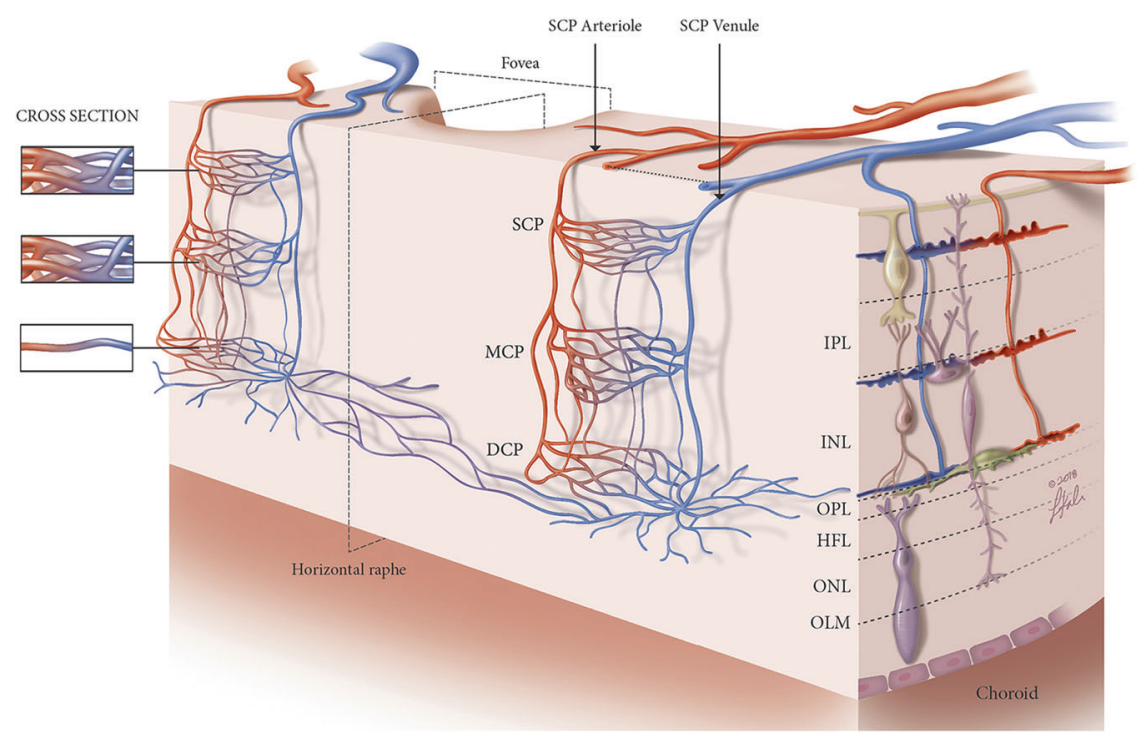

Fig. 1 Illustration of 3D spatial relationships and vascular connectivity between the macular capillary plexuses. Figure taken from Nesper and Fawzi [26]. Large superficial capillary plexus (SCP) arterioles (red) and venules (blue) supply and drain each of the three capillary plexuses in a largely parallel arrangement. The capillary plexuses are further interconnected by anastomoses. Additional capillaries traverse the horizontal raphe and provide series connections between different beds of the deep capillary plexus (DCP). Other abbreviations: HFL Henle fibre layer; INL inner nuclear layer; IPL inner plexiform layer; MCP middle capillary plexus; OLM outer limiting membrane; ONL outer nuclear layer; OPL outer plexiform layer.

peripapillary capillaries are an additional, fourth vascular layer found superficially around the optic nerve [24]. While several models have been proposed to describe the connectivity and relationships between the SCP, MCP, and DCP [25], most suggest that the three layers are supplied and drained by the large SCP arterioles and venules in a hybrid model with parallel capillary beds connected by anastomoses between the layers and limited series connections between DCP vessels crossing the horizontal raphe, forming an overall highly interconnected network (Fig. 1) [26-28]. This predominantly parallel arrangement of capillary beds would provide distinct arterial supply and venous drainage for each layer, facilitating their function as independent neurovascular units $[29,30]$.

Custom OCTA segmentation protocols can separate the anatomic MCP from the SCP and DCP, enabling analysis of the individual plexuses (Fig. 2). And indeed, OCTA studies of human eyes in light and dark adaptation experiments support that the SCP, MCP, and DCP are differentially regulated. In healthy eyes in the light, the MCP and DCP constrict while the SCP dilates to supply the metabolically active inner retinal cells (Fig. 3, top left) [31]. Conversely, in the dark, the MCP and DCP dilate, increasing blood flow to the underlying photoreceptors to meet increased metabolic demand (Fig. 3, top right) [31, 32].

Furthermore, OCTA has provided an important adjunct to methods like fundoscopic vessel imaging in evaluating the effects of perturbations and pathologic changes, including diabetes, on retinal neurovascular coupling responses [33, 34]. For example, caffeine causes constriction of the MCP in the dark and delays the light adaptation response, suggesting dysregulation that may precipitate transient ischemia [35]. On the other hand, acute hyperglycaemia reverses the neurovascular coupling response in healthy eyes entirely, leading to a paradoxical constriction of the DCP in the dark, and MCP and DCP dilation in the light (Fig. 3, bottom row) [36].

Recently, our group showed that the dark adaptation response is deranged in diabetics with pre-clinical or mild non-proliferative retinopathy (NPDR) [37]. Compared to healthy controls, diabetic subjects without DR and those with early NPDR showed reversed responses to light adaptation with SCP constriction and MCP dilation, similar to the reversed response seen in healthy eyes exposed to acute hyperglycaemia. These dark adaptation data corroborate the results of older studies using other imaging modalities that showed abnormal neurovascular responses and diminished sensitivity to dark adaptation in the diabetic retina $[33,34,38-40]$. The disruption of normal light responses in acute hyperglycaemia and early diabetes suggests hyperglycaemia may contribute to subclinical ischemia by altering the normal oxygen gradient in the retina during times of shifting metabolic demand. Because the DCP is also thought to contribute to the oxygen and vascular supply of the underlying photoreceptor cells [41, 42], reversal of the normal dark dilation and light constriction response of this layer may exacerbate photoreceptor stress and inflammation [43], and contribute to early diabetic neural dysfunction as well $[44,45]$.

\section{OCTA changes with DR progression}

Vascular changes on OCTA have been shown to correlate with DR severity. Many cross-sectional studies have characterized OCTA parameters across DR severity, finding similar general trends. Overall, vessel density decreases [46-52] and FAZ area increases $[53,54]$ as DR severity increases, likely due to capillary loss and diminished perfusion within existing vessels (Fig. 4). Notably, one recent study comparing the size of the FAZ on structural OCT to the FAZ area on OCTA suggested that the apparent increase in OCTA FAZ area seen in increasing DR severity may actually result from nonperfusion rather than from vessel loss, since these ghost vessels were still detectable on highly averaged structural OCT images [55]. Our group used a multivariate model that combined multiple parameters from OCTA (vessel density and FAZ metrics) to distinguish diabetic eyes without DR, NPDR, and PDR with high specificity and sensitivity [56].

Despite an overall agreement on the trends of capillary loss with DR progression, the relative extent of vessel density loss in the different capillary layers is still debated. Some studies suggest DCP vessel density changes are more significant $[48,51]$, while others suggest SCP vessel density is better correlated with DR severity $[46,52]$. Further complicating the interpretation of these results is that these studies do not consider the MCP separately. Most commercial OCTA systems use a built-in image segmentation that divides the retinal capillaries into superficial and deep 

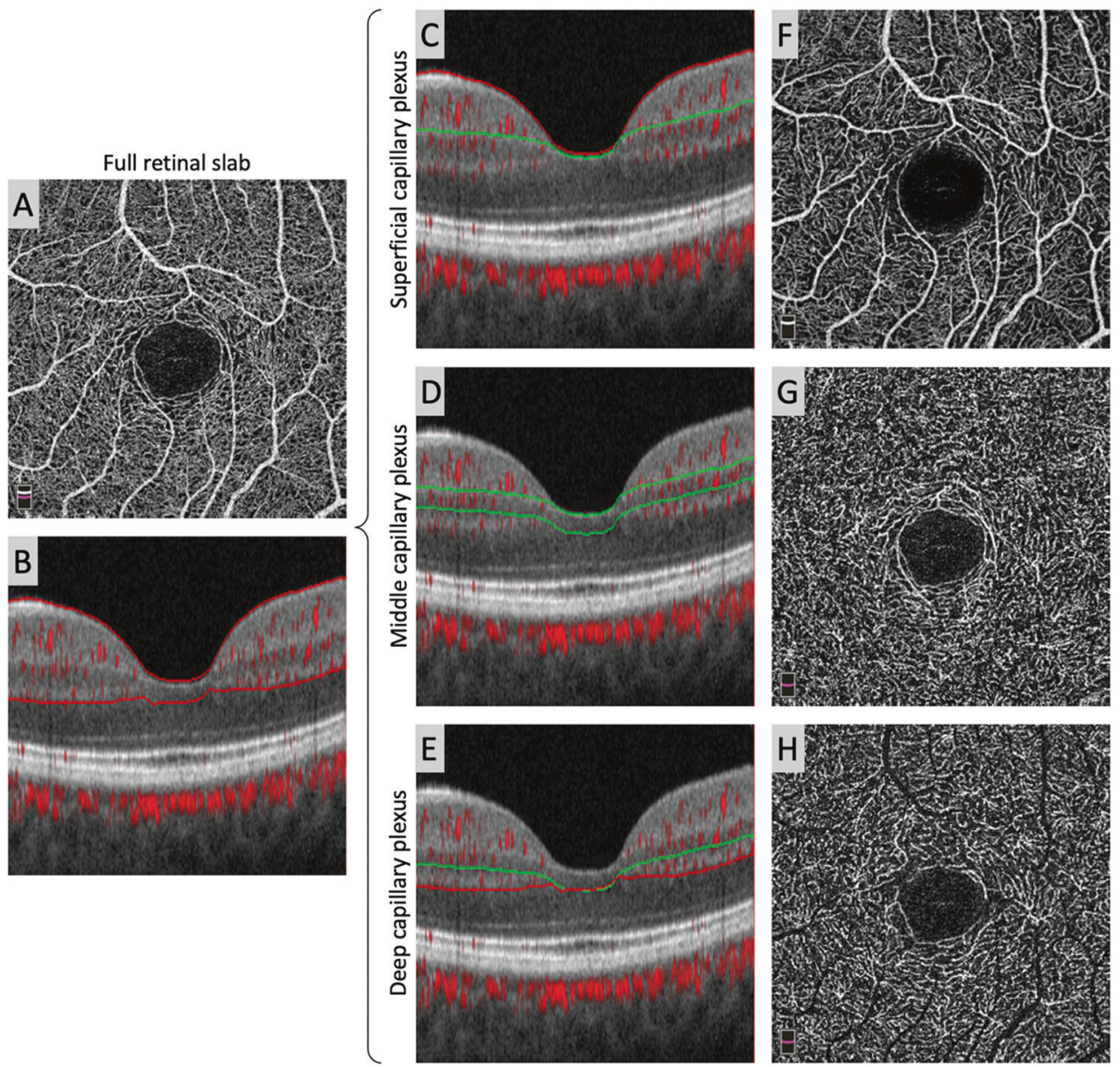

Fig. 2 Segmentation of OCTA slabs enables isolation and analysis of three capillary layers in the macula. A En face full retinal slab from a healthy eye and B structural OCT B-scan with overlaid flow signal. C Segmentation and F corresponding en face slab, respectively, for the superficial; D, G middle; and $\mathbf{E}$, $\mathbf{H}$ deep capillary plexuses.

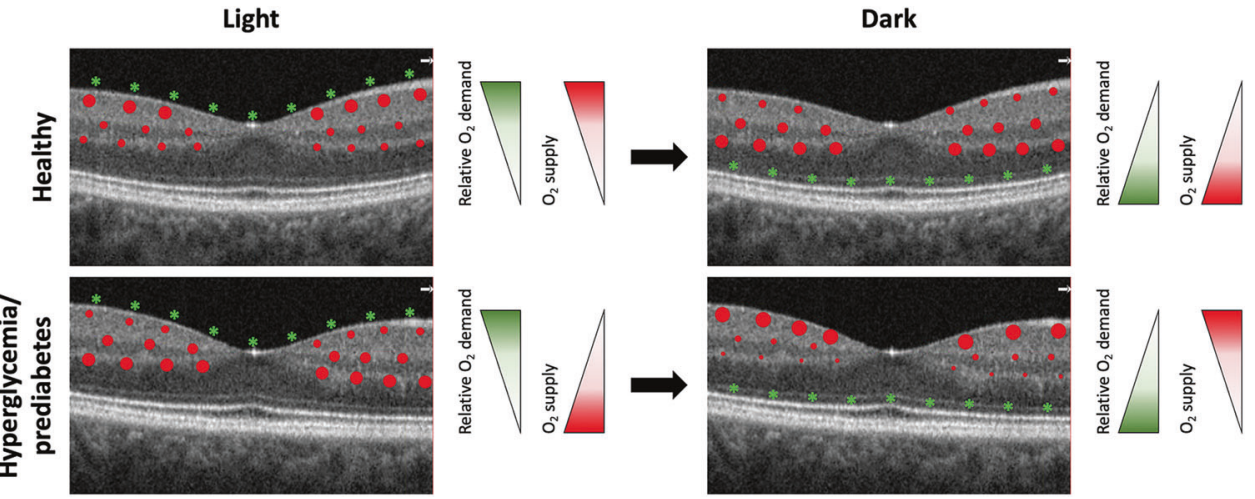

Fig. 3 Hyperglycaemic and early diabetic eyes show reversal of neurovascular coupling. Vessel cross-sections are shown in red. Areas of increased metabolic demand are indicated by green asterisks. In healthy eyes in the light (top left), superficial vessels are relatively dilated to meet increased demand by inner retinal cells. In the dark (top right), superficial vessels constrict while the deep layers dilate to meet photoreceptor demand. However, in acute hyperglycaemia and in preclinical DR, these neurovascular coupling responses are reversed, with deep layer dilation in the light (bottom left) and superficial dilation in the dark (bottom right). These mismatched gradients of oxygen demand (green wedges) and supply (red wedges) may contribute to subclinical ischemia in early diabetes.

layers, with the anatomic MCP incorporated into or split between the layers [57-59]. Studies that evaluated the MCP separately in DR suggest that MCP changes tend to follow those of the DCP $[21,58,60]$. It is generally thought that analyzing the MCP separately can increase the sensitivity for detecting early nonperfusion, especially within the MCP and DCP [21].
OCTA can also visualize the more subtle vascular changes in different sub-stages of NPDR, an important area of research as we strive to risk-stratify these subjects. For example, mild and moderate NPDR are clinically associated with a lower one-year risk of progression to PDR (5-15\%) and visual complications compared to severe NPDR (52-72\%)[4]. Two recent studies have 

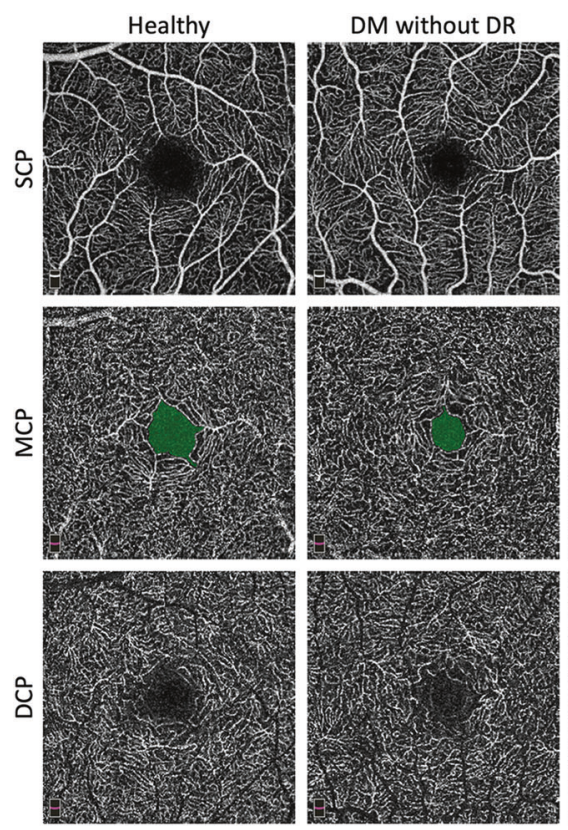
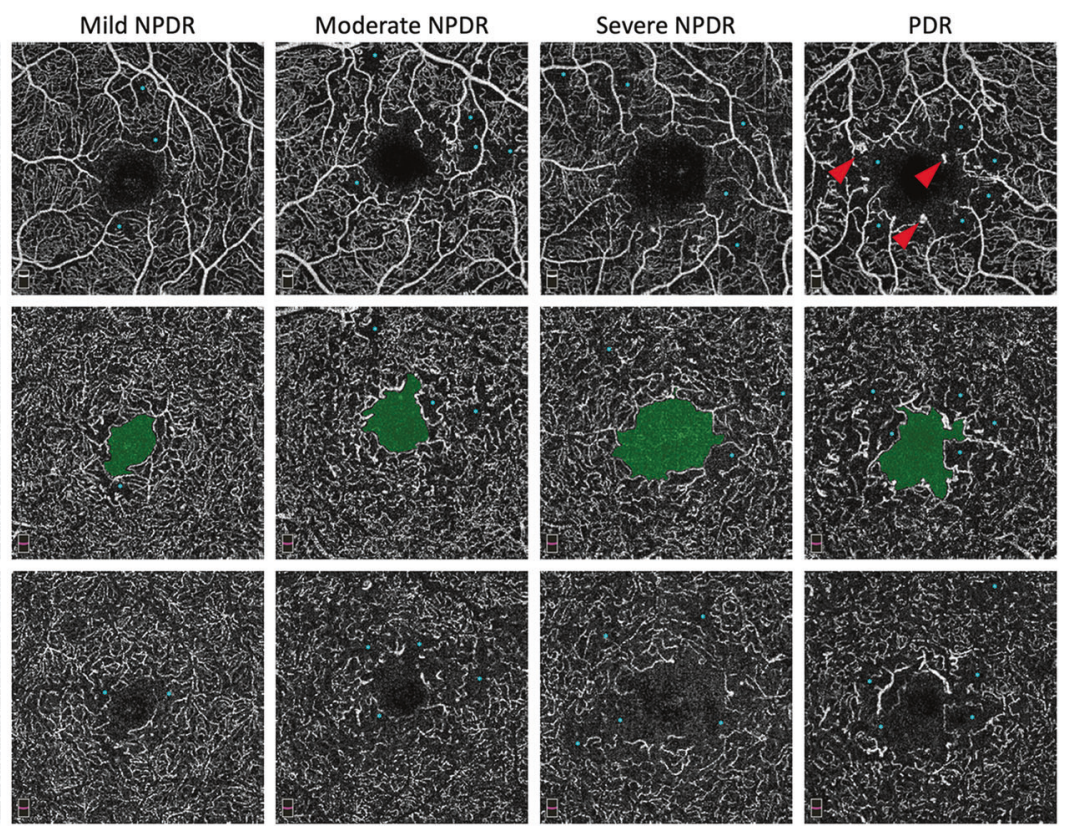

Fig. 4 OCTA imaging shows decreasing vessel density and increasing foveal avascular zone (FAZ) size with DR severity. Superficial (SCP; top row), middle (MCP; middle row), and deep capillary plexuses (DCP; bottom row) in representative healthy eyes, diabetics without DR (DM without DR), mild non-proliferative DR (Mild NPDR), moderate NPDR, severe NPDR, and proliferative DR (PDR). Capillary nonperfusion results in FAZ (green) enlargement with worsening DR. Areas of capillary dropout (cyan asterisks) can be seen with the onset of NPDR and increase in size as DR becomes more severe. Upregulation of vascular endothelial growth factor (VEGF) in response to worsening ischemia in PDR leads to worsening dilation of telangiectatic vessels (red arrows) and neovascularization.

suggested that severe NPDR eyes show significantly decreased SCP vessel density compared to moderate NPDR, which could be very useful to stratify patients who are at higher risk for visual complications [61, 62]. Decreased vessel densities are also seen in eyes with mild NPDR compared to those without DR, suggesting OCTA may also be useful in screening for DR onset [61, 62].

\section{Estimating blood flow from OCTA}

OCTA images not only provide vascular structural parameters like vessel density and FAZ metrics, but also may reflect the rate of vascular flow, which has been explored in several studies [63, 64]. Adjusted flow index (AFI) is a parameter that represents a normalized pixel intensity from OCTA images, which has been shown through in vitro experiments to correlate linearly with flow velocities within a physiologic range [63]. This would suggest that OCTA not only yields a proxy for blood flow velocity in retinal vessels [63], but could also be used to monitor disease-related changes that impact blood flow [64]. AFI has been suggested to show differential changes in each retinal capillary layer with progressive DR severity. Generally, AFI in the MCP and DCP decreases as DR severity worsens [48, 51, 58]. However, AFI changes in the SCP have shown somewhat conflicting results, though the overall trend is for increased AFI. Two studies found an initial increase in SCP AFI in diabetic eyes without DR compared to healthy controls, but no significant trends in more severe stages $[48,58]$. Another study found an overall significant increase in SCP AFI with advancing DR severity, but no significant difference between diabetics without DR and healthy controls [61].

Taken together, the existing data would suggest that as DR severity worsens, blood flow in the MCP and DCP decreases in the face of unchanged or even increased SCP flow. These apparently opposite changes in the different layers may reflect autoregulatory dysfunction of the retinal capillaries, consistent with the impaired neurovascular coupling known to occur in diabetic eye disease. Another potential explanation could be that the dilated telangiectatic vessels in the SCP have lower resistance, which then potentially exacerbates low flow at the MCP/DCP via a "steal phenomenon" [65].

\section{Characterizing OCTA changes in pre-clinical DR and the utility of adaptive optics}

While clinically apparent retinopathy is associated with measurable changes in vascular parameters like vessel density and to a lesser extent, flow indices, the nature of early structural microvascular changes in diabetic eyes without clinical manifestations of DR is poorly understood. Although OCTA dark adaptation studies and other tests of neurovascular coupling do suggest functional impairment in retinal blood flow autoregulation in diabetic eyes without DR, such tests are time-consuming, not widely available, and generate data that require more complex interpretation, restricting their use to smaller research studies.

While a few studies have suggested increased FAZ area in diabetic eyes without DR compared to healthy controls $[66,67]$, many others found no significant differences [48, 68-70]. Even in healthy eyes, FAZ area shows substantial inter-individual variability, which may limit detection of disease-related changes $[71,72]$. Mixed results have also been seen for vessel density, with some studies suggesting reduced macular vessel density $[66,73]$ and others finding no differences in the pre-clinical stages of DR $[69,70,74]$.

Recently, it has been suggested that capillary density may actually be increased in the region surrounding the FAZ $(200 \mu \mathrm{m}$ annulus) in diabetic subjects without DR. This has been suggested to occur as a response to subclinical ischemia leading to capillary dilation or recruitment of formerly non-perfused capillaries [75]. This finding of increased central perfusion, as well as the increased SCP AFI that has been reported in diabetic eyes without DR $[48,58]$, suggest that blood flow and thus perfusion may initially increase in pre-clinical DR, although additional studies with larger sample sizes and further standardization of flow quantification will be important to investigate flow and vessel changes in preclinical DR. 

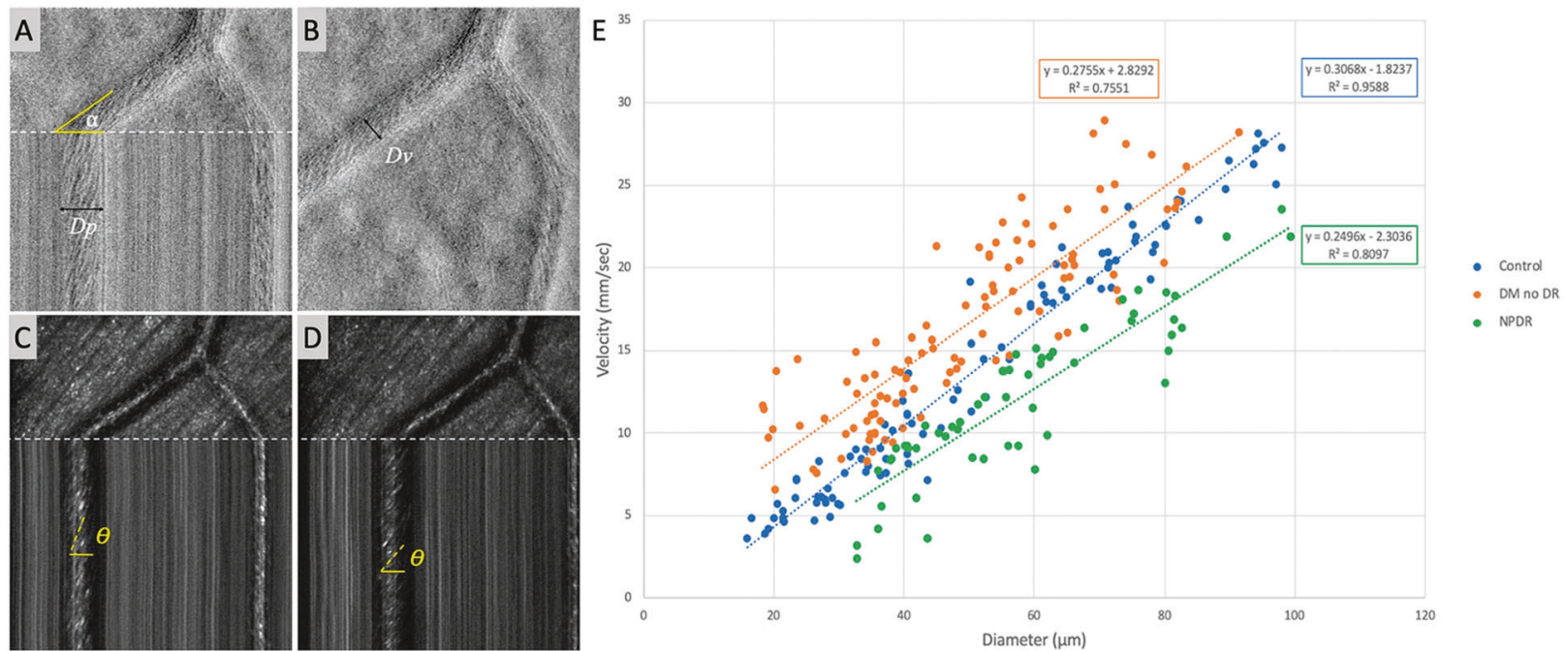

Fig. 5 AOSLO-XT imaging suggests blood flow is increased in diabetic eyes without DR (DM no DR). Figure adapted from Palochak et al. [81]. In XT imaging, a single cross-section is scanned repeatedly in split-detection (A, B) and confocal (C, D) modes. Vessel angle relative to the detector $(\alpha)$ and vessel cross-section width $(D p)$ are used to determine vessel luminal diameter $(D v)$, which, combined with angle of erythrocyte translocation between scans $(\theta)$, is used to calculate blood flow rate. Smaller $\theta$ will indicate faster flow. E On average, DM no DR eyes show higher blood flow rates over all vessel diameters than healthy controls (Control) or eyes with early non-proliferative DR (NPDR).

Other retinal imaging modalities like adaptive optics (AO) may provide a more precise assessment of flow alterations in preclinical DR. AO imaging uses a wavefront sensor and deformable lens to correct the optical aberrations in the eye and increase imaging resolution [76]. AO has been combined with various techniques including OCT [77] and scanning laser ophthalmoscopy (SLO) [78] to visualize retinal structures such as the photoreceptor mosaic by resolving the individual cells [79]. AOSLO can also resolve individual erythrocytes or erythrocyte trains within retinal vessels, a property that has been utilized in a further modification of AOSLO, XT scanning (AOSLO XT) [80]. In AOSLO XT, a single blood vessel cross-section is scanned repeatedly over time (Fig. 5A-D). While the vessel walls and other retinal structures remain static, the erythrocytes will translocate across the frame over time as blood flows through the vessel. This movement is visualized as "streaks". The blood flow rate is then calculated based on the rate of erythrocyte movement over time and the diameter of the blood vessel.

Using AOSLO XT to quantify flow in healthy eyes and compare them to diabetic eyes with no DR or with mild NPDR, we recently found that blood flow velocity increases in diabetic subjects without DR, but decreases in those with NPDR relative to healthy controls (Fig. 5E) [81]. These trends persisted across a range of vessel diameters from medium $(15-60 \mu \mathrm{m})$ to large $(60-100 \mu \mathrm{m})$ -sized retinal vessels. Currently, the clinical utility of AOSLO is limited due to equipment cost and the complicated imaging protocol, which may be difficult due to diabetic vision changes; and restriction of visualization to a single point in a single blood vessel in each session [76, 80, 82]. Nonetheless, these preliminary findings also support increased blood flow in diabetic eyes prior to DR onset, which may represent a compensatory mechanism to increase perfusion in response to subclinical ischemia or a secondary effect from the release of vasoactive mediators [8385]. These results also corroborate the results of older Dopplerbased studies showing normal to increased flow in early diabetic eyes [86-88]. This flow increase may be analogous to the glomerular hyperfiltration stage seen in diabetic kidneys prior to the development of nephropathy [89].

\section{Monitoring treatment response}

Quantitative changes in vessel parameters not only track with the severity of DR but are also associated with response to treatment.
OCTA parameters such as vessel tortuosity and fractal dimension, which evaluate the geometric arrangement of the capillary networks, worsen as DR severity increases, becoming more tortuous and less complex, respectively [90-92]. Increasing tortuosity is associated with the pro-angiogenic effects of VEGF, which promotes the formation of new disorganized and leaky blood vessels as well as dilation and leakage of existing vessels [93-95]. Interestingly, these anomalous parameters appear to reverse following pan-retinal photocoagulation (PRP) [90]. This laser treatment is thought to effect its benefit by ablating peripheral photoreceptors, lowering the overall retinal metabolic demand, and decreasing the pro-angiogenic drive, which then promotes regression of neovascularization and vascular telangiectasias while reducing high-risk PDR characteristics.

Furthermore, using AFI as a surrogate metric for blood flow, PRP treatment was shown to also improve macular blood flow, while paradoxically increasing macular nonperfused areas in treated PDR (Fig. 6) [96]. This finding is consistent with the application of PRP to the peripheral retina, with secondary constriction of the peripheral circulation due to increased flow of oxygen from the choroid to the inner retina. As a result, retinal blood flow that would otherwise be divided between peripheral and macular circulation is primarily diverted to the macula, thereby improving macular perfusion. Along with improved macular circulation, there is remodelling of the macular capillaries, with "pruning" of some of the less functional macular capillaries, along with increased flow in the healthier ones. PRP is also associated with increased FAZ circularity, suggesting the restoration of flow to previously nonperfused vessels in the perifovea, also consistent with the model of PRP flow diversion from the peripheral to macular circulation [97]. Finally, OCTA may also be used to non-invasively monitor the size of neovascularization in response to anti-VEGF treatment [98].

\section{Overall model}

Based on the findings of advanced retinal imaging in recent years, we present a model of DR progression that encompasses many of the quantifiable changes identified in the studies described above (Fig. 7). Chronic hyperglycaemia as seen in pre-diabetes leads to abnormal neurovascular coupling of the retinal capillaries, resulting in aberrant responses that contribute to overall subclinical retinal ischemia. In diabetes prior to the onset of DR, blood flow initially increases, mainly in the SCP, and can partially 

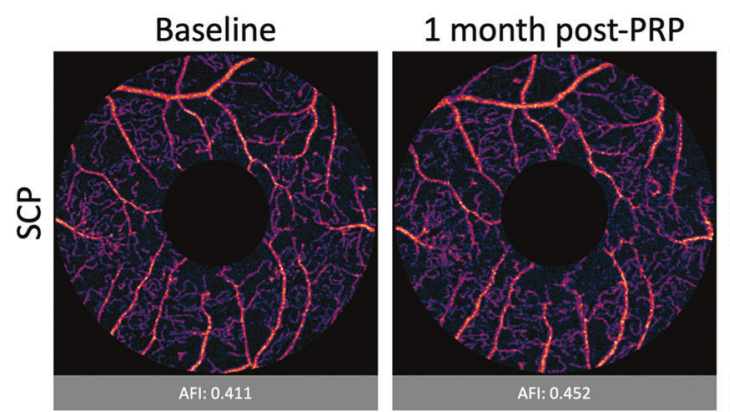

\section{6 months post-PRP}
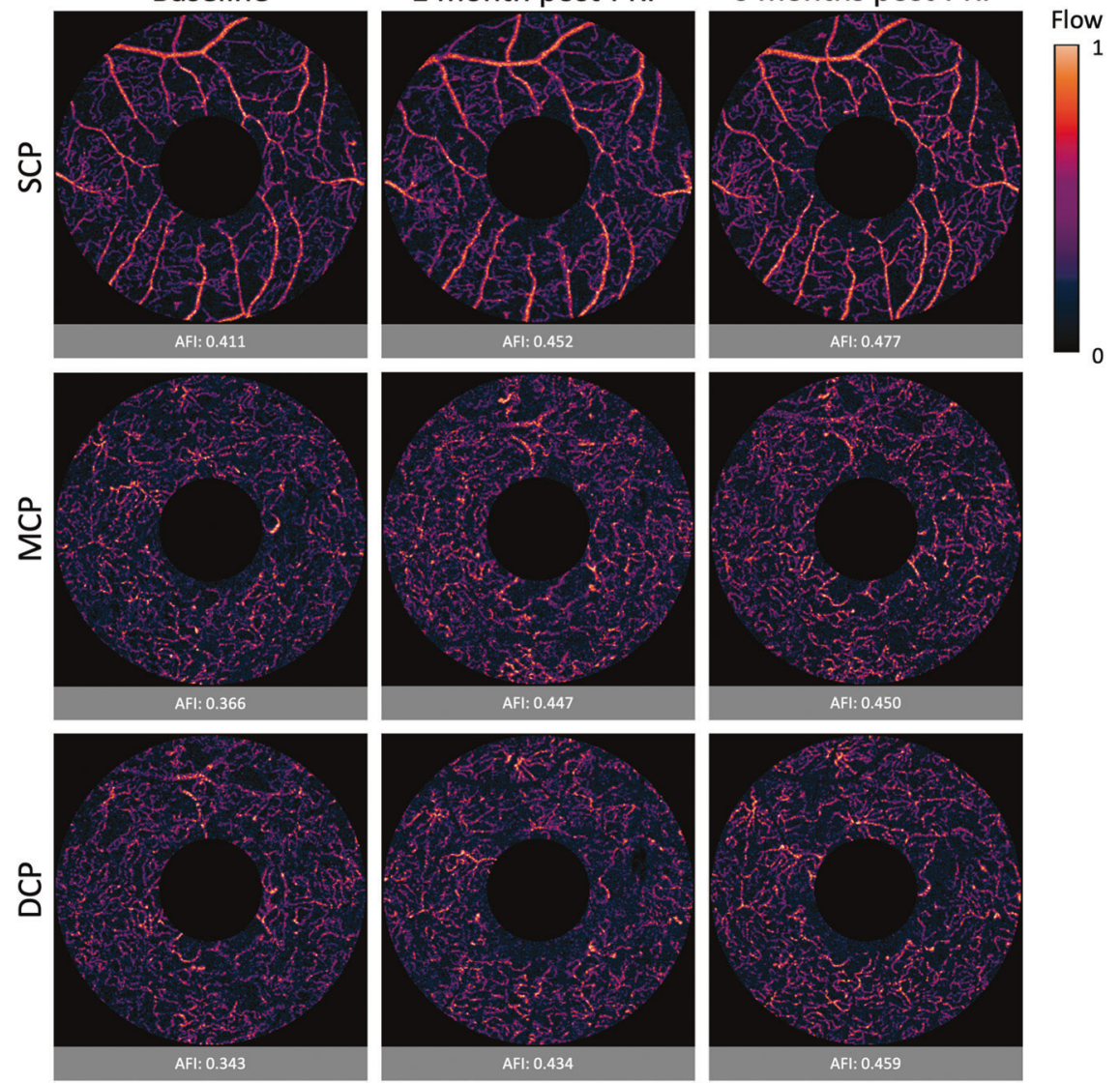

Fig. 6 PRP treatment leads to stable improvement in macular flow in eyes with PDR. Representative example of an eye with PDR before (left column), 1 month after (middle column), and 6 months after PRP laser treatment (right column). Adjusted flow index (AFI) increases compared to baseline and remains stable in the superficial (SCP; top row), middle (MCP; middle row), and deep (DCP; bottom row) capillary plexuses following PRP.

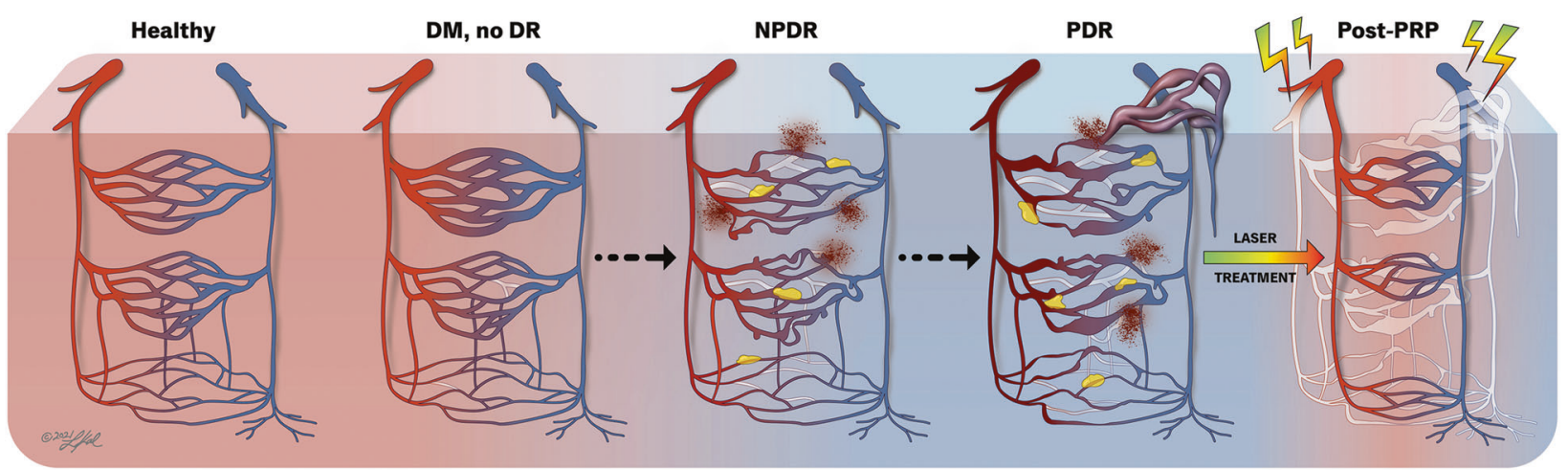

Fig. 7 Proposed model of DR progression based on findings from advanced retinal imaging. Increasing ischemia is indicated by blue gradient background. Hyperglycaemia in early diabetes (DM, no DR) contributes to abnormal neurovascular responses leading to subclinical ischemia, which promotes a compensatory increase in blood flow from the superficial vessels. Failure of these compensatory mechanisms leads to decreased blood flow, which along with continued capillary injury (nonperfused vessels in white) leads to the appearance of clinical DR (NPDR). Leakage of protein- and lipid-rich fluid from damaged capillaries contributes to the formation of hard exudates (yellow). Progression to PDR occurs when a threshold of upregulated VEGF is reached in response to worsening ischemia, promoting neovascularization that further exacerbates ischemia, especially in the deep layers. By decreasing the photoreceptor metabolic demand and the VEGF load, PRP leads to regression of the leaky neovascularization in the peripheral circulation and peripheral vasoconstriction, which promotes remodelling and diverts blood flow from the retinal periphery to the macula, normalizing macular circulation parameters and reducing the ischemic drive.

compensate for this chronic low-level ischemia. With the continued closure of the capillaries, however, there is a failure of these compensatory mechanisms, with decreased blood flow and the appearance of clinical retinopathy in NPDR, as well as progressive loss of perfused vessel density exacerbating ischemia, especially in the deeper layers. As NPDR severity worsens and vessel dropout progresses, PDR develops when a threshold of VEGF upregulation is reached. This VEGF also contributes to SCP 
telangiectatic changes that further divert flow from an increasingly depleted MCP and DCP circulation. The telangiectatic SCP capillaries continue to preferentially shunt flow into the lowerresistance dilated SCP at the expense of the higher-resistance MCP and DCP, feeding a vicious cycle of worsening ischemia. Treatments like PRP, which reduce peripheral photoreceptor demand and promote peripheral vasoconstriction and decreased peripheral blood flow, help to increase overall macular perfusion of the macula and contribute to relative normalization of blood flow.

\section{Summary and future directions}

Advanced retinal imaging modalities like OCTA and AOSLO have great potential, not just for clinical staging and monitoring of $D R$, but also as important tools for studying in vivo structural and functional changes in diabetic eye disease. Combining these imaging modalities with emerging computational tools such as artificial intelligence and machine learning algorithms may offer new insight and improve the clinical workflow, not only for screening large populations of patients but potentially also for predicting the course of DR in individual eyes $[50,99,100]$. Further study of advanced retinal imaging in larger and more diverse populations, over longitudinal time courses, and in a wider variety of treatments and experimental settings will help inform our understanding of diabetic eye disease and expand the utility of these devices in a wider range of clinical applications. Overall, these tools will enhance our ability to monitor and predict highrisk individuals who may benefit from new treatment algorithms. Ultimately, pharmacologic treatments aimed at improving blood flow and capillary health would greatly benefit DR, and these therapies could be a great match for the advanced vascular imaging tools that we have outlined here.

\section{REFERENCES}

1. Lee R, Wong TY, Sabanayagam C. Epidemiology of diabetic retinopathy, diabetic macular edema and related vision loss. Eye Vis. 2015;2:17.

2. Saeedi P, Petersohn I, Salpea P, Malanda B, Karuranga S, Unwin N, et al. Global and regional diabetes prevalence estimates for 2019 and projections for 2030 and 2045: results from the International Diabetes Federation Diabetes Atlas, 9 (th) edition. Diabetes Res Clin Pr. 2019;157:107843.

3. Nentwich MM, Ulbig MW. Diabetic retinopathy-ocular complications of diabetes mellitus. World J Diabetes. 2015;6:489-99.

4. Aiello LM. Perspectives on diabetic retinopathy. Am J Ophthalmol. 2003;136:122-35.

5. He F, Xia X, Wu XF, Yu XQ, Huang FX. Diabetic retinopathy in predicting diabetic nephropathy in patients with type 2 diabetes and renal disease: a meta-analysis. Diabetologia. 2013;56:457-66.

6. Kawasaki R, Tanaka S, Tanaka S, Abe S, Sone H, Yokote K, et al. Risk of cardiovascular diseases is increased even with mild diabetic retinopathy: the Japan diabetes complications study. Ophthalmology. 2013;120:574-82.

7. Oh IK, Kim SW, Oh J, Lee TS, Huh K. Inflammatory and angiogenic factors in the aqueous humor and the relationship to diabetic retinopathy. Curr Eye Res. 2010;35:1116-27.

8. Funatsu $H$, Yamashita $H$, Noma H, Mimura T, Nakamura S, Sakata K, et al. Aqueous humor levels of cytokines are related to vitreous levels and progression of diabetic retinopathy in diabetic patients. Graefes Arch Clin Exp Ophthalmol. 2005;243:3-8.

9. Chen $\mathrm{H}$, Zhang $\mathrm{X}$, Liao N, Wen F. Assessment of biomarkers using multiplex assays in aqueous humor of patients with diabetic retinopathy. BMC Ophthalmol. 2017;17:176.

10. Simo R, Sundstrom JM, Antonetti DA. Ocular Anti-VEGF therapy for diabetic retinopathy: the role of VEGF in the pathogenesis of diabetic retinopathy. Diabetes Care. 2014;37:893-9.

11. Hammes HP, Feng $Y$, Pfister F, Brownlee M. Diabetic retinopathy: targeting vasoregression. Diabetes. 2011;60:9-16.

12. Curtis TM, Gardiner TA, Stitt AW. Microvascular lesions of diabetic retinopathy: clues towards understanding pathogenesis? Eye. 2009;23:1496-508.

13. Mizutani M, Kern TS, Lorenzi M. Accelerated death of retinal microvascular cells in human and experimental diabetic retinopathy. J Clin Invest. 1996;97:2883-90.
14. Stitt AW, Curtis TM, Chen M, Medina RJ, McKay GJ, Jenkins A, et al. The progress in understanding and treatment of diabetic retinopathy. Prog Retin Eye Res. 2016;51:156-86.

15. Gardiner TA, Archer DB, Curtis TM, Stitt AW. Arteriolar involvement in the microvascular lesions of diabetic retinopathy: implications for pathogenesis. Microcirculation. 2007;14:25-38.

16. Duh EJ, Sun JK, Stitt AW. Diabetic retinopathy: current understanding, mechanisms, and treatment strategies. JCI Insight. 2017;2:e93751.

17. Jia Y, Tan O, Tokayer J, Potsaid B, Wang Y, Liu JJ, et al. Split-spectrum amplitudedecorrelation angiography with optical coherence tomography. Opt Express. 2012;20:4710-25.

18. Chua J, Sim R, Tan B, Wong D, Yao X, Liu X, et al. Optical coherence tomography angiography in diabetes and diabetic retinopathy. J Clin Med. 2020;9:7-14.

19. Gao SS, Jia Y, Zhang M, Su JP, Liu G, Hwang TS, et al. Optical coherence tomography angiography. Invest Ophthalmol Vis Sci. 2016;57:OCT27-36.

20. Park JJ, Soetikno BT, Fawzi AA. Characterization of the middle capillary plexus using optical coherence tomography angiography in healthy and diabetic eyes. Retina. 2016;36:2039-50.

21. Hwang TS, Zhang $M$, Bhavsar $K$, Zhang $X$, Campbell JP, Lin $P$, et al. Visualization of 3 distinct retinal pexuses by projection-resolved optical coherence tomography angiography in diabetic retinopathy. JAMA Ophthalmol. 2016;134:1411-9.

22. Spaide RF, Klancnik JM Jr, Cooney MJ. Retinal vascular layers imaged by fluorescein angiography and optical coherence tomography angiography. JAMA Ophthalmol. 2015;133:45-50.

23. Chan G, Balaratnasingam C, Yu PK, Morgan WH, McAllister IL, Cringle SJ, et al. Quantitative morphometry of perifoveal capillary networks in the human retina. Invest Ophthalmol Vis Sci. 2012;53:5502-14.

24. Tan PE, Yu PK, Balaratnasingam C, Cringle SJ, Morgan WH, McAllister IL, et al. Quantitative confocal imaging of the retinal microvasculature in the human retina. Invest Ophthalmol Vis Sci. 2012;53:5728-36.

25. Garrity ST, Paques M, Gaudric A, Freund KB, Sarraf D. Considerations in the understanding of venous outflow in the retinal capillary plexus. Retina. 2017;37:1809-12.

26. Nesper PL, Fawzi AA. Human parafoveal capillary vascular anatomy and connectivity revealed by optical coherence tomography angiography. Invest Ophthalmol Vis Sci. 2018;59:3858-67.

27. Campbell JP, Zhang M, Hwang TS, Bailey ST, Wilson DJ, Jia Y, et al. Detailed vascular anatomy of the human retina by projection-resolved optical coherence tomography angiography. Sci Rep. 2017;7:42201.

28. An D, Yu P, Freund KB, Yu DY, Balaratnasingam C. Three-dimensional characterization of the normal human parafoveal microvasculature using structural criteria and high-resolution confocal microscopy. Invest Ophthalmol Vis Sci. 2020;61:3.

29. Leahy C, Radhakrishnan H, Weiner G, Goldberg JL, Srinivasan VJ. Mapping the $3 \mathrm{D}$ connectivity of the rat inner retinal vascular network using OCT angiography. Invest Ophthalmol Vis Sci. 2015;56:5785-93.

30. Kornfield TE, Newman EA. Regulation of blood flow in the retinal trilaminar vascular network. J Neurosci. 2014;34:11504-13.

31. Nesper PL, Lee HE, Fayed AE, Schwartz GW, Yu F, Fawzi AA. Hemodynamic response of the three macular capillary plexuses in dark adaptation and flicker stimulation using optical coherence tomography angiography. Invest Ophthalmol Vis Sci. 2019;60:694-703.

32. Braun RD, Linsenmeier RA, Goldstick TK. Oxygen consumption in the inner and outer retina of the cat. Invest Ophthalmol Vis Sci. 1995;36:542-54.

33. Dorner GT, Garhofer G, Huemer KH, Riva CE, Wolzt M, Schmetterer L. Hyperglycemia affects flicker-induced vasodilation in the retina of healthy subjects. Vis Res. 2003;43:1495-500.

34. Kappelgaard P, Holfort SK, Klefter ON, Larsen M. Retinal vessel diameter changes in relation to dark adaptation and acute hyperglycemia. J Ophthalmol. 2018;2018:7064359.

35. Zhang YS, Lee HE, Kwan CC, Schwartz GW, Fawzi AA. Caffeine delays retinal neurovascular coupling during dark to light adaptation in healthy eyes revealed by optical coherence tomography angiography. Invest Ophthalmol Vis Sci. 2020;61:37.

36. Kwan CC, Lee HE, Schwartz G, Fawzi AA. Acute hyperglycemia reverses neurovascular coupling during dark to light adaptation in healthy subjects on optical coherence tomography angiography. Invest Ophthalmol Vis Sci. 2020;61:38.

37. Zhang YS, Mucollari I, Kwan CC, Dingillo G, Amar J, Schwartz GW, et al. Reversed neurovascular coupling on optical coherence tomography angiography is the earliest detectable abnormality before clinical diabetic retinopathy. J Clin Med. 2020;9:3523.

38. Lim LS, Ling LH, Ong PG, Foulds W, Tai ES, Wong E, et al. Dynamic responses in retinal vessel caliber with flicker light stimulation in eyes with diabetic retinopathy. Invest Ophthalmol Vis Sci. 2014;55:5207-13. 
39. Lim LS, Ling LH, Ong PG, Foulds W, Tai ES, Wong TY. Dynamic responses in retinal vessel caliber with flicker light stimulation and risk of diabetic retinopathy and its progression. Invest Ophthalmol Vis Sci. 2017;58:2449-55.

40. Henson DB, North RV. Dark adaptation in diabetes mellitus. Br J Ophthalmol. 1979;63:539-41.

41. Scarinci F, Nesper PL, Fawzi AA. Deep retinal capillary nonperfusion is associated with photoreceptor disruption in diabetic macular ischemia. Am J Ophthalmol. 2016;168:129-38.

42. Birol G, Wang S, Budzynski E, Wangsa-Wirawan ND, Linsenmeier RA. Oxygen distribution and consumption in the macaque retina. Am J Physiol Heart Circ Physiol. 2007;293:H1696-704.

43. Du Y, Veenstra A, Palczewski K, Kern TS. Photoreceptor cells are major contributors to diabetes-induced oxidative stress and local inflammation in the retina. Proc Natl Acad Sci USA. 2013;110:16586-91.

44. Harrison WW, Bearse MA Jr, Ng JS, Jewell NP, Barez S, Burger D, et al. Multifocal electroretinograms predict onset of diabetic retinopathy in adult patients with diabetes. Invest Ophthalmol Vis Sci. 2011;52:772-7.

45. Abcouwer SF, Gardner TW. Diabetic retinopathy: loss of neuroretinal adaptation to the diabetic metabolic environment. Ann NY Acad Sci. 2014;1311:174-90.

46. Durbin MK, An L, Shemonski ND, Soares M, Santos T, Lopes M, et al. Quantification of retinal microvascular density in optical coherence tomographic angiography images in diabetic retinopathy. JAMA Ophthalmol. 2017;135:370-6.

47. Kim AY, Chu Z, Shahidzadeh A, Wang RK, Puliafito CA, Kashani AH. Quantifying microvascular density and morphology in diabetic retinopathy using spectraldomain optical coherence tomography angiography. Invest Ophthalmol Vis Sci. 2016;57:OCT362-70.

48. Nesper PL, Roberts PK, Onishi AC, Chai H, Liu L, Jampol LM, et al. Quantifying microvascular abnormalities with increasing severity of diabetic retinopathy using optical coherence tomography angiography. Invest Ophthalmol Vis Sci. 2017;58:BIO307-BIO15.

49. Agemy SA, Scripsema NK, Shah CM, Chui T, Garcia PM, Lee JG, et al. Retinal vascular perfusion density mapping using optical coherence tomography angiography in normals and diabetic retinopathy patients. Retina. 2015;35:2353-63.

50. Alam M, Zhang Y, Lim Jl, Chan RVP, Yang M, Yao X. Quantitative optical coherence tomography angiography features for objective classification and staging of diabetic retinopathy. Retina. 2020;40:322-32.

51. Sambhav K, Abu-Amero KK, Chalam KV. Deep capillary macular perfusion indices obtained with OCT angiography correlate with degree of nonproliferative diabetic retinopathy. Eur J Ophthalmol. 2017;27:716-29.

52. Li L, Almansoob S, Zhang P, Zhou YD, Tan Y, Gao L. Quantitative analysis of retinal and choroid capillary ischaemia using optical coherence tomography angiography in type 2 diabetes. Acta Ophthalmol. 2019;97:240-6.

53. Takase N, Nozaki M, Kato A, Ozeki H, Yoshida M, Ogura Y. Enlargement of foveal avascular zone in diabetic eyes evaluated by en face optical coherence tomography angiography. Retina. 2015;35:2377-83.

54. Choi W, Waheed NK, Moult EM, Adhi M, Lee B, De Carlo T, et al. Ultrahigh speed swept source optical coherence tomography angiography of retinal and choriocapillaris alterations in diabetic patients with and without retinopathy. Retina. 2017;37:11-21.

55. Lynch G, Romo JSA, Linderman R, Krawitz BD, Mo S, Zakik A, et al. Within-subject assessment of foveal avascular zone enlargement in different stages of diabetic retinopathy using en face OCT reflectance and OCT angiography. Biomed Opt Express. 2018;9:5982-96.

56. Ashraf M, Nesper PL, Jampol LM, Yu F, Fawzi AA. Statistical model of optical coherence tomography angiography parameters that correlate with severity of diabetic retinopathy. Invest Ophthalmol Vis Sci. 2018;59:4292-8.

57. Fawzi AA. Consensus on optical coherence tomographic angiography nomenclature: do we need to develop and learn a new language? JAMA Ophthalmol. 2017;135:377-8.

58. Onishi AC, Nesper PL, Roberts PK, Moharram GA, Chai H, Liu L, et al. Importance of considering the middle capillary plexus on OCT angiography in diabetic retinopathy. Invest Ophthalmol Vis Sci. 2018;59:2167-76.

59. Spaide RF, Fujimoto JG, Waheed NK. Image artifacts in optical coherence tomography angiography. Retina. 2015;35:2163-80.

60. Zhang M, Hwang TS, Dongye C, Wilson DJ, Huang D, Jia Y. Automated quantification of nonperfusion in three retinal plexuses using projection-resolved optical coherence tomography angiography in diabetic retinopathy. Invest Ophthalmol Vis Sci. 2016;57:5101-6.

61. Ong JX, Kwan CC, Cicinelli MV, Fawzi AA. Superficial capillary perfusion on optical coherence tomography angiography differentiates moderate and severe nonproliferative diabetic retinopathy. PLoS One. 2020;15:e0240064.
62. Ashraf M, Sampani K, Clermont A, Abu-Qamar O, Rhee J, Silva PS, et al. Vascular density of deep, intermediate, and superficial vascular plexuses are differentially affected by diabetic retinopathy severity. Invest Ophthalmol Vis Sci. 2020;61:53.

63. Tokayer J, Jia Y, Dhalla AH, Huang D. Blood flow velocity quantification using split-spectrum amplitude-decorrelation angiography with optical coherence tomography. Biomed Opt Express. 2013;4:1909-24.

64. Ploner SB, Moult EM, Choi W, Waheed NK, Lee B, Novais EA, et al. Toward quantitative optical coherence tomography angiography: visualizing blood flow speeds in ocular pathology using variable interscan time analysis. Retina. 2016;36:S118-S26. Suppl 1

65. Bek T. Diameter changes of retinal vessels in diabetic retinopathy. Curr Diab Rep. 2017;17:82.

66. Dimitrova G, Chihara E, Takahashi H, Amano H, Okazaki K. Quantitative retinal optical coherence tomography angiography in patients with diabetes without diabetic retinopathy. Invest Ophthalmol Vis Sci. 2017;58:190-6.

67. de Carlo TE, Chin AT, Bonini Filho MA, Adhi M, Branchini L, Salz DA, et al. Detection of microvascular changes in eyes of patients with diabetes but not clinical diabetic retinopathy using optical coherence tomography angiography. Retina. 2015;35:2364-70.

68. Lu Y, Simonett JM, Wang J, Zhang M, Hwang T, Hagag AM, et al. Evaluation of automatically quantified foveal avascular zone metrics for diagnosis of diabetic retinopathy using optical coherence tomography angiography. Invest Ophthalmol Vis Sci. 2018;59:2212-21.

69. Goudot MM, Sikorav A, Semoun O, Miere A, Jung C, Courbebaisse B, et al. Parafoveal OCT angiography features in diabetic patients without clinical diabetic retinopathy: a qualitative and quantitative analysis. J Ophthalmol. 2017;2017:8676091.

70. Thompson IA, Durrani AK, Patel S. Optical coherence tomography angiography characteristics in diabetic patients without clinical diabetic retinopathy. Eye. 2019;33:648-52.

71. Shahlaee A, Pefkianaki M, Hsu J, Ho AC. Measurement of foveal avascular zone dimensions and its reliability in healthy eyes using optical coherence tomography angiography. Am J Ophthalmol. 2016;161:50-5. e1

72. Samara WA, Say EA, Khoo CT, Higgins TP, Magrath G, Ferenczy $S$, et al. Correlation of foveal avascular zone size with foveal morphology in normal eyes using optical coherence tomography angiography. Retina. 2015; 35:2188-95.

73. Cao D, Yang D, Huang Z, Zeng Y, Wang J, Hu Y, et al. Optical coherence tomography angiography discerns preclinical diabetic retinopathy in eyes of patients with type 2 diabetes without clinical diabetic retinopathy. Acta Diabetol. 2018;55:469-77.

74. Dai Y, Zhou H, Chu Z, Zhang Q, Chao JR, Rezaei KA, et al. Microvascular changes in the choriocapillaris of diabetic patients without retinopathy investigated by swept-source OCT angiography. Invest Ophthalmol Vis Sci. 2020;61:50.

75. Rosen RB, Andrade Romo JS, Krawitz BD, Mo S, Fawzi AA, Linderman RE, et al. Earliest evidence of preclinical diabetic retinopathy revealed using optical coherence tomography angiography perfused capillary density. Am J Ophthalmol. 2019;203:103-15.

76. Merino D, Loza-Alvarez P. Adaptive optics scanning laser ophthalmoscope imaging: technology update. Clin Ophthalmol. 2016;10:743-55.

77. Zhang $\mathrm{Y}$, Rha J, Jonnal R, Miller D. Adaptive optics parallel spectral domain optical coherence tomography for imaging the living retina. Opt Express. 2005;13:4792-811.

78. Merino D, Dainty C, Bradu A, Podoleanu AG. Adaptive optics enhanced simultaneous en-face optical coherence tomography and scanning laser ophthalmoscopy. Opt Express. 2006;14:3345-53.

79. Pircher M, Zawadzki RJ, Evans JW, Werner JS, Hitzenberger CK. Simultaneous imaging of human cone mosaic with adaptive optics enhanced scanning laser ophthalmoscopy and high-speed transversal scanning optical coherence tomography. Opt Lett. 2008;33:22-4.

80. Zhong Z, Petrig BL, Qi X, Burns SA. In vivo measurement of erythrocyte velocity and retinal blood flow using adaptive optics scanning laser ophthalmoscopy. Opt Express. 2008;16:12746-56.

81. Palochak CMA, Lee HE, Song J, Geng A, Linsenmeier RA, Burns SA, et al. Retinal blood velocity and flow in early diabetes and diabetic retinopathy using adaptive optics scanning laser ophthalmoscopy. J Clin Med. 2019:8:1165.

82. Godara P, Dubis AM, Roorda A, Duncan JL, Carroll J. Adaptive optics retinal imaging: emerging clinical applications. Optom Vis Sci. 2010;87:930-41.

83. Newman EA. Functional hyperemia and mechanisms of neurovascular coupling in the retinal vasculature. J Cereb Blood Flow Metab. 2013;33:1685-95.

84. Kito K, Tanabe K, Sakata K, Fukuoka N, Nagase K, lida M, et al. Endotheliumdependent vasodilation in the cerebral arterioles of rats deteriorates during acute hyperglycemia and then is restored by reducing the glucose level. J Anesth. 2018;32:531-8. 
85. Williams SB, Goldfine AB, Timimi FK, Ting HH, Roddy MA, Simonson DC, et al. Acute hyperglycemia attenuates endothelium-dependent vasodilation in humans in vivo. Circulation. 1998;97:1695-701.

86. Grunwald JE, DuPont J, Riva CE. Retinal haemodynamics in patients with early diabetes mellitus. Br J Ophthalmol. 1996;80:327-31.

87. Wang Y, Fawzi A, Tan O, Gil-Flamer J, Huang D. Retinal blood flow detection in diabetic patients by Doppler Fourier domain optical coherence tomography. Opt Express. 2009;17:4061-73.

88. Wang Y, Fawzi AA, Varma R, Sadun AA, Zhang X, Tan O, et al. Pilot study of optical coherence tomography measurement of retinal blood flow in retinal and optic nerve diseases. Invest Ophthalmol Vis Sci. 2011;52:840-5.

89. Tonneijck L, Muskiet MH, Smits MM, van Bommel EJ, Heerspink HJ, van Raalte $\mathrm{DH}$, et al. Glomerular hyperfiltration in diabetes: mechanisms, clinical significance, and treatment. J Am Soc Nephrol. 2017;28:1023-39.

90. Fayed AE, Abdelbaki AM, El Zawahry OM, Fawzi AA. Optical coherence tomography angiography reveals progressive worsening of retinal vascular geometry in diabetic retinopathy and improved geometry after panretinal photocoagulation. PLoS One. 2019;14:e0226629.

91. Sasongko MB, Wong TY, Nguyen TT, Cheung CY, Shaw JE, Wang JJ. Retinal vascular tortuosity in persons with diabetes and diabetic retinopathy. Diabetologia 2011;54:2409-16.

92. Lee $H$, Lee $M$, Chung $H$, Kim HC. Quantification of retinal vessel tortuosity in diabetic retinopathy using optical coherence tomography angiography. Retina. 2018;38:976-85.

93. Paques M, Krivosic V, Girmens JF, Giraud C, Sahel J, Gaudric A. Decreased venous tortuosity associated with resolution of macular edema after intravitreal injection of triamcinolone. Retina. 2005;25:1099-101.

94. Hartnett ME, Martiniuk D, Byfield G, Geisen P, Zeng G, Bautch VL. Neutralizing VEGF decreases tortuosity and alters endothelial cell division orientation in arterioles and veins in a rat model of ROP: relevance to plus disease. Invest Ophthalmol Vis Sci. 2008;49:3107-14.

95. Prasanna P, Bobba V, Figueiredo N, Sevgi DD, Lu C, Braman N, et al. Radiomicsbased assessment of ultra-widefield leakage patterns and vessel network architecture in the PERMEATE study: insights into treatment durability. $\mathrm{Br} \mathrm{J}$ Ophthalmol. 2020.

96. Fawzi AA, Fayed AE, Linsenmeier RA, Gao J, Yu F. Improved macular capillary flow on optical coherence tomography angiography after panretinal photocoagulation for proliferative diabetic retinopathy. $\mathrm{Am} J$ Ophthalmol. 2019;206:217-27.

97. Faghihi $H$, Riazi-Esfahani $H$, Khodabande A, Khalili Pour E, Mirshahi A, Ghassemi $F$, et al. Effect of panretinal photocoagulation on macular vasculature using optical coherence tomography angiography. Eur J Ophthalmol. 2020; 31:1877-1884.

98. He F, Yu W. Longitudinal neovascular changes on optical coherence tomography angiography in proliferative diabetic retinopathy treated with panretinal photocoagulation alone versus with intravitreal conbercept plus panretinal photocoagulation: a pilot study. Eye. 2020;34:1413-8.
99. Ting DSW, Cheung CY, Lim G, Tan GSW, Quang ND, Gan A, et al. Development and validation of a deep learning system for diabetic retinopathy and related eye diseases using retinal images from multiethnic populations with diabetes. JAMA. 2017;318:2211-23.

100. Walton OBT, Garoon RB, Weng CY, Gross J, Young AK, Camero KA, et al. Evaluation of automated teleretinal screening program for diabetic retinopathy. JAMA Ophthalmol. 2016;134:204-9.

\section{ACKNOWLEDGEMENTS}

The authors thank Lauren Kalinoski MS, for her contributions to the artwork of Figs. 1 and 7. This research was supported by NIH grant R01 EY31815. Research instrument support was provided by Optovue, Inc., Fremont, California, USA. The funders had no role in study design, data collection, and analysis, decision to publish, or preparation of the manuscript.

\section{AUTHOR CONTRIBUTIONS}

JXO was responsible for identifying and interpreting relevant studies, writing the review article, and creating figures. AAF generated the overall concept and provided feedback to revise drafts.

\section{FUNDING}

AAF was funded in part by NIH grant R01 EY31815. The funders had no role in study design, data collection, and analysis, decision to publish, or preparation of the manuscript. Research instrument support was provided by Optovue, Inc., Fremont, California, USA with no involvement in research funding, design, recruitment, or manuscript production.

\section{COMPETING INTERESTS}

Dr. Fawzi has served as a consultant for Regeneron Pharmaceuticals, Genentech, and Boehringer Ingelheim. These groups had no role in study design, data collection, and analysis, decision to publish, or preparation of the manuscript. Janice Ong declares no potential competing interests.

\section{ADDITIONAL INFORMATION}

Correspondence and requests for materials should be addressed to Amani A. Fawzi.

Reprints and permission information is available at http://www.nature.com/reprints

Publisher's note Springer Nature remains neutral with regard to jurisdictional claims in published maps and institutional affiliations. 